accuracy. He has two eyes as well as two ears. A bone, a lead pencil, and a twisted piece of paper sometimes double the chance of success. Object teaching is the natural method; demonstrate the facts where practicable.

2. Avoid controversies with the cross-examiner. The war is unprofitable because unequal; he can strike when the surgeon is down; his last turn with court and jury comes when the medical man is no longer an active participant in the case.

3. Keep sweet. He who loses his temper loses his cause. Questions are frequently asked for the express purpose of irritating the surgeon; an exhibition of anger is the very result desired, and the first ruffling of composure is welcomed as a successful assault on the enemy's works.

4. Talk slowly; take time to answer questions fully. Minds untrained in medical lore can not follow and comprehend professional explanations at the same rate of speed as assistants at a clinic.

One of the most common methods of perplexing a witness and neutralizing the effect of his testimony is to fire volleys of rapid questions at him. Half finished, hastily given answers create erroneous and contradictory impressions; hence the necessity for deliberation throughout expert testimony, and an utter disregard of attempts to undo the surgeon through undue haste.

5. Answer the question asked. $\mathrm{He}$ who volunteers information or explanation digs a pit into which the cross-examiner shall shortly but surely tumble him. In the infrequent instances where "yes" and "no" will not suffice as answers, an explanation of the crossexaminer's ingenious verbal trap will be developed by questions of opposing counsel on re-direct examination, or will be permitted upon request to the court for opportunity to make the matter clear.

6. Answer briefly. The best approved judicial proceedings are in accord with the petitions of those Indian Territory negroes who habitually besought the Judge of all hearts to grant them "long prayers and short answers." The plaintiff in his complaint or declaration "ever prays," and the defendant in his answer "will ever pray," at considerable length and with much technical repetition, but that surgeon most helps his cause who in his testimony gives short answers and shuns technicalities.

7. Say, "I do not know." The surgeon who knows everything is clay in the hands of the cross-examiner, and malformed or formless clay in the verdict of the jurymen.

8. Do not discount the medical information possessed by the cross-examiner. Thousands of lawyers beside Lord Eldon have studied medicine before reading law. Lacking a medical education, the lawyer prepares his case by repeated consultations with leading physicians, by reading articles in medical journals and by careful examination of the accredited medical authorities.

As was recently said by a distinguished New York attorney in addressing the graduating class of a great medical school, "The medical profession differs from the legal in the fact that the physician is not expected to know law, but the lawyer must have a familiar and comprehensive knowledge of medicine."

JOINT AIMS AND AOHIEVEMENTS OF MEDICINE AND LAIV. \begin{tabular}{l|l} 
LAW. & $\begin{array}{l}\text { aged expectantly, than the lesions of the } \\
\text { Personally the doctor may never need the lawyer's }\end{array}$ \\
are now subjected to surgical treatment.
\end{tabular} services, though the attorney is sure to require the physician's ministrations; but professionally the advocate who fights against oppression is the blood comrade of the physician who battles against death. Together they have warred for the rights of the poor, the helpless and the despised; together they have defied kings and corporations. When, in the dark ness of the middle ages, all other good seemed lost, the doctor and the barrister stood alone as mouthpieces and interpreters of the common people-soli. tary champions of liberty and justice, unconquered and unterrified by kings. In our own day, through their joint efforts, boards of health have been organized, State and Government inspection of water and food products introduced, reformatories and insane asylums reconstructed, prison conditions ameliorated and upheaved, and many other great reforms instituted.

HIGH MORAL STANDARD DEMANDED OF PHYGICIAN AND ATTORNEY.

There can be no human liberty without law; there can be no healthy human liberty without medicine; the tyranny of rulers and the tyranny of disease alike reduce men to slavery. "Noblesse oblige," said the old French king, and he who breaks these shackles from humanity must regard neither men nor money, neither position nor power. Leprosy and smallpox are less to be dreaded than the moral contagion of dishonor. Whether in clinic or court room, whether beside the sickbed or before the bar, temporizing, policy, the "necessity" which Pitt called " the argument of tyrants" and "the creed of slaves," are only weights he he has laid aside. His rule of life is the Supreme Law enunciated by the Great Healer: "Ye shall know the truth, and the truth shall make you free;" and his earthly ambitions are summed up in. Wolsey's counsel to Cromwell:

"Be just and fear not.

Let all the ends thou aims' $t$ at be thy country's, Thy God's, and truth's, then if thou fall'st, O Cromwell, Thou fall'st a blessed martyr."

-Shakspeare, Henry VIII; Act III: Sc. 2.

\section{THE SURGERY OF THE BRAIN.}

Address delivered before the Austin Flint Medical Society, Cloar Lake, Ia., July 20, 1897 .

BY ALBERT I. BOUFFLEUR, B.S., M.D.,

Professor of Anatomy and Clinical Surgery, Northwestern University Woman's Medical School; Demonstrator of Operative Surgery, Rush Medical College; Attending Surgeon Cook County Hospital, etc.

CHICAGO, ILL.

The surgery of the brain and its membranes has within the last two or three years received more merited attention than during the preceding decade, when the great surgical world spent a large portion of its energy on that important class of diseases which are found in the abdomen and which are now so generally subjected to surgical treatment.

All subjects which directly affect the life of an individual are of special interest to the conscientious physician and certainly there is no class of diseases which are more obscure in their symptomatology, more erratic in their course, more discouraging in their treatment or more uniformly fatal, when managed expectantly, than the lesions of the brain, which
are now subjected to surgical treatment. 
The peculiar anatomic conditions in which the or. area for smell and taste. In addition, later investigagan under consideration is placed renders the oppor. tion seems to have established the fact that there is a tunity for careful observation of very rare occurrence, center or centers for muscular sense and to have deterwhile the location of the vital centers in the lower mined its probable location.

brain precludes all thorough and systematic investi-

1. The motor-area is located in the convolutions gation upon man. It is only by clinical observation bounding the fissure of Rolando and those adjacent then, that even a fairly comprehensive knowledge of thereto; those of one hemisphere control the movethe location of the foci of physiologic action and of ments of the opposite side of the body, while the gendiseased processes has been adduced. The patholo- eral arrangement of the centers is in inverse order to gists have discovered inflammatory, suppurative and the location of the parts controlled; the highest cenadventitious processes in the brain for many years, ters being for the leg and foot while the lowest are and they have also been able to classify the various for the face, etc. As most people are right handed lesions which are found therein, and even to finally differentiate between types and degrees thereof, but until these lesions could be clinically located with a fair degree of certainty, the local treatment was of necessity purely empirical.

Experimental research on animals and clinical cbservation with postmortem findings in man, led to the discovery of motor centers and later of centers for special sense, and more recently closer studies have outlined the cortical centers for different varieties and degrees of physiologic manifestations. With the location of the physiologic centers came the possibility of the clinical diagnosis and location of disease processes. As these processes were subjected to surgical treatment in other parts of the body with a large degree of success, it was but natural that this field was considered an inviting, though admittedly difficult one, for the exercise of the surgeon's skill and dexterity.

Operations upon the brain were so uniformly fatal in pre-antiseptic days that the surgery of the brain was, by common consent, limited to emergency work. The protection afforded by antiseptics and asepsis has rendered the cranial cavity safely approachable, while the various improvements in the methods of trephining and means for its execution have rendered much of the cranial contents readily accessible.

Successful cerebral surgery depends upon three essential elements, viz., the diagnosis of the character of the disease, its anatomic location and the proper operative technique. The purpose of this paper is to briefly present the nature and diagnosis of cerebral lesions, to emphasize the importance of cerebral localization and to discuss the advisability of subjecting our patients with cerebral diseases to operations for the relief of their troubles, and if an operation is deemed advisable, how it should be performed to obtain the best results. The diagnosis of most of the conditions which we will consider should be made or confirmed by a neurologist when possible before being surgically treated.

\section{CEREBRAL LOCALIZATION.}

In considering this subject it is well to remember that while there are points or small areas of the brain in which the reaction to stimulation is most marked, the area of lesser reaction extends in most instances far over into other areas so that the various centers or areas overlap each other. This fact explains the observation that when a primary anatomic center only is removed the function ascribed to that area may not be wholly paralyzed. It is also recognized that these areas are not confined to any single convolution or even lobe, but may be located in two or more.

The areas which are now generally conceded to be definitely located are the motor-sensory area, the speech area, the visual area, the auditory area and the that it is loceted in the inferior parietal region but 
there have been cases in which destruction of the motor speech area has caused its loss - agraphia.

3. The visual area or sight center is located in the cuneus and cortex of the occipital lobe. As each lobe receives impulses from half of both eyes, a lesion causes hemianopsia, half blindness in both eyes, and the field of blindness is on the opposite side of the lesion, according to Starr, while the Charcot theory is that a lesion of one center causes total blindness of the opposite eye.

4. The auditory area is located in the first or superior and second or middle temporal convolutions. Each ear being connected with both hemispheres, the effect of a unilateral lesion is only partial deafness and is usually not noticed. Bilateral lesions cause total deafness.

5. The area for sensations of smell and taste is located at the tip of the temporal lobe on its inner or under surface. Each lobe is related to the organs of both sides and hence unilateral lesions cause only diminished function.

The location of the psychic center is undecided. Lesions in the frontal lobes have been manifested by little else than mental symptoms, while lesions have been found, postmortem, in the frontal lobes of subjects who have been free from any mental symptoms.

The symptomatology of lesions limited to the basal ganglia is unknown. It is only when the lesion en. croaches upon the internal capsule or some radiating or connecting fibers that symptoms are produced.

Lesions in the pons and medulla give rise to irritation or destruction of the cranial nerve centers of the particular region involved.

The cerebellum controls the equilibrium of the body and, therefore, disturbance of its structure is productive of vertigo and staggering.

The works of Ferrier, Horsley, Henschen, Knapp's table in "Strümpell's Practice," Starr and others present the subject more in detail. The diagnostician who contemplates work in this class of diseases should master at least this much of what is known about cerebral localization while the operator should, in addition, be able to locate the areas on the surface of the skull.

The subjects which will briefly claim our attention th are trephining for fractures of the skull, intra-cranial hemorrhage, suppuration and tumors, epilepsy, insanity, imbecility, hydrocephalus, removal of Gasserian ganglion and for pain.

Fractures of the skull.-This subject is considered because it is so frequently attended by evidences of injuries to the brain and its coverings and especially as the expectant treatment thereof is a potent factor in producing those pathologic conditions-hemorrhage, inflammation, abscess, etc.-for which we are called upon to operate subsequently.

Fractures are divided into simple, compound, com. plicated and depressed. A simple fracture is one unattended by a wound: a compound, one connected with a wound; a complicated, one accompanied by extensive damage to overlying soft parts, or positive injury to the brain or its membranes or to the vessels, or by an infection of the external wound, and a depressed fracture is one in which one fragment is lower than normal and which may or may not be cottended by symptoms of cerebral compression. A depressed is a complicated fracture and it may be either simple or compound. Frequently, as in gunshot wounds, we find the three latter divisions associated.
In the absence of a wound, the diagnosis of a fracture is frequently purely conjectural. The nature and direction of the operating force should always be carefully considered. The presence of a well defined swelling with sharp borders due to an exudate or blood clot in the scalp or beneath the periosteum may readily lead one to believe that a depressed fracture is present when, in fact, there is only a circumscribed contusion. Likewise the possibility of congenital and other defects in bone should be considered.

When there is a wound it is, as a rule, easy to determine the condition of the bone although it may be necessary to enlarge the opening to definitely settle the question.

In every case in which there is doubt, the scalp should be sufficiently incised to fully expose the area and admit of a thorough digital and ocular examination. Two preliminary conditions are however requisite: 1, the area should be thoroughly shaved and rendered positively aseptic; 2 , the operator should be prepared to perform any operation which the local conditions indicate. There has been much discussion as to the treatment of skull fractures, which has resulted in the formulation of many sets of rules to govern the surgeon. It, however, remains a fact that even those of largest experience are frequently at a loss to know whether to operate or not in a given case. In compound fractures some sort of an operation is always indicated for the excision of bruised and lacerated tissues, the removal of splinters of bone and foreign bodies, the elevation of depressed bone, controlling of hemorrhage, and at least for the proper closure of the wound. In bullet wounds it is generally conceded to be best to leave the missile undisturbed, unless it can be definitely located in a superficial position, the injury to the skull being treated as a compound fracture. The question of drainage must be decided upon the indications of each case. The frequency of the occurrence of traumatic epilepsy and other serious secondary effects, from the old plan of treating fractures of the skull and the fact that when properly performed the operation of trephining is practically without mortality, has led to the establishment of a more advanced plan of treatment in this class of cases. Even in simple fractures the patient should be subjected to operation when there is depression of a fragment or where there are evidences of injury to the intracranial tissues. The full extent of the damage can be determined only by this method, while anything short of it does not permit of adequate treatment where so much is in doubt. If mor'e fractures were thoroughly exposed, more depressions would be detected, and if more depressed fractures were elevated, more blood clots turned out and more spicula of bone removed by trephining, as a primary operation, we would have less epilepsy and other sequelæ which are so frequently traceable to a trauma of the head.

In all cases of fracture of the skull the general treatment should comprise complete physiologic rest, free intestinal and renal elimination and, if necessary, the application of ice to the head.

Intracranial hemorrhage.-In cases when progressive symptoms of compression supervene upon the receipt of a trauma, which are attended by the manifestation of stimulation (irritation) or paralysis of a motor area the diagnosis of hemorrhage is probably justifiable. The gradual development of stupor followed by coma and stertorous breathing, a slow pulse, 
a dilated pupil and a rise of temperature to less than 103 degrees makes the symptomatology complete. The differentiation between extra-dural and intradural hemorrhage is not sufficiently determined to be of practical value.

Statistics demonstrate the demand for operation in cases where any considerable amount of hemorrhage has probably occurred. Park gives Weisman's reports in which of 147 cases treated expectantly, 131 died, while of 110 treated by operation only 30 proved fatal. Selection of cases would undoubtedly improve these figures. It should be remembered that most hemorrhages occur from the middle meningeal artery and that they are most frequently extra-dural. Where the focal symptoms point to compression of the opposite side the operation should be performed on the side presenting the symptoms.

Intra-cranial suppuration.-This class comprises abscess, suppurative leptomeningitis and thrombophlebitis. Abscess of the brain may be traumatic or non-traumatic. The former variety may develop either soon after a traumatism or years later. Where a wound has been present it is usually indicative of its having been the seat of prolonged suppuration. The nontraumatic variety is usually the result of the extension of an infection from the middle ear, although it may follow an orbital or nasal infection, or may be of metastatic origin. One of the strangest features of cerebral abscess is the great variance of the time of its occurrence. The symptoms of an acute abscess may subside or none may appear, and yet, from some unknown cause, months or years afterward, the symptoms of abscess suddenly develop. Nature has probably succeeded in confining it to a definite region and if that happens to be in an unimportant area of the brain no symptoms may be occasioned thereby, until the protecting wall gives way, when all evidences of an encephalitis are manifested. In making a diag. nosis we should always look for a traumatism or a primary suppurating focus, as upon its presence or absence may rest the differentiation from cerebral tumor. In addition to the headache, vomiting, vertigo and symptoms of pressure there may be fever or a subnormal temperature with the probable absence of ocular evidences. In latent abscesses the symptoms are especially obscure and its diagnosis often impossible. Abscess following middle ear disease is of frequent occurrence and deserves much more attention than is usually accorded to it and demands radical treatment. Suppurative otitis may be followed by a leptomengitis or a septic thrombo-phlebitis and abscess. If the perforation is upward the lesion will be located in the temporo-sphenoidal region ; if backward, in the cerebellar region, while if by an extension of the process through the mastoid veins it will cause a thrombo-phlebitis of the sigmoid sinus.

The intimate connection of the mastoid veins with the lining of the mastoid cells and the sinus render infection by this route especially likely to occur. The differential diagnosis of the various forms of intracranial infection is not always possible since two or more varities are likely to be associated. Jansen has given this subject special study and states that pyemic fever with repeated chills following a suppurative otitis are indications of sinus thrombosis and if these are accompanied by symptoms of vertigo, restlessness, nausea, rigidity of neck, stupor, etc., operation is demanded. ("Ann. Surg." Vol. xxxiii.) The statistics are very favorable to radical operative treatment.
The treatment of suppuration within the cranium should be upon the same principles as in other regions of the body, with special indications for prompt and radical measures, since the prognosis of expectantly treated intra.cranial suppuration is so uniformly bad. When abscesses can be located they should be exposed and thoroughly disinfected and drained, preferably with gauze packing. If the abscess is in the temporal region, Von Bergman ("Ann. Surgery," Vol. xxiii.) advises operating so as to expose the tympanum and then extending the section as the conditions may indicate. Likewise in sinus thrombosis the sigmoid fossa can be easily reached after exposing the mastoid cells. When thrombosis of the lateral sinus is present the condition of the interal jugular vein should be determined, and if it is thrombosed it should be exposed in the neck, and, after ligation below the location of thrombosis, it should be opened and the sinus and vein thoroughly emptied and disinfected. When the symptoms point to a suppurative leptomeningitis the indication is for thorough drainage, the same as for suppuration in any other serous cavity.

The cranium should be trephined below the superior curved line of the occiput and also above the posterior part of the zygoma (Park). These openings will admit of free irrigation and drainage.

Intra-cranial tumors. - The most common varieties of brain tumors are sarcoma, glioma, tubercle, secondary carcinoma, syphiloma and cysts, in the order named. The symptoms of tumor partake of the same general character as those of all lesions which increase intracranial pressure. Persistent headache, intractable vomiting and optic neuritis, with evidences of focal stimulation or destruction and the absence of a primary suppuration focus, are strongly indicative of cerebral tumor, and should differentiate it from other lesions. Our present knowledge of the symptomato. logy of tumors is not sufficient to enable us to differentiate except in a general way. The smaller, harder, more circumscribed and less vascular the growth the better the prospects of recovery. They are more frequently located in the base and the cerebellum in children, while in adults the cerebral cortex is fortunately most frequently involved.

About one-third of all brain tumors are located in the basal ganglia and, therefore, situated in a surgically inaccessible region. Nearly one-fourth of the tumors given in "Starr's Tabulation" were located in the cerebellum, whence tumors are excised with the greatest difficulty. One-tenth of the number were multiple in character. The remaining tumors were located in accessible regions. Of these many did not present the necessary focal symptoms, while others were of such size or character as to have precluded their removal. It is then definitely understood that only about 6 per cent. of all brain tumors can be successfully removed by operation. The fact that the diagnosis as to character and location of the lesion is frequently obscure, and that when made it is frequently erroneous, coupled with the inoperability of such a large percentage makes the present field of operative treatment of brain tumors very limited and the prospects for relief in general poor indeed.

Cases of syphilitic and even non-syphilitic growths have been relieved by antisyphilitic treatment, therefore, all cases of tumor of the brain should be subjected to a course of heroic treatment for at least three 
months before operation is resorted to. Notwithstanding the limits of the operation and the recognized dangers of the procedure, we must remember that cases which are not relieved by medication gradually grow worse, until life becomes one of mental degeneration and often one of great misery.

In nearly one hundred recent cases collected by Starr the results of the operations have been successful in 46 per cent. The result of operation in cerebral tumors is more favorable than in those located in the cerebellum. Even in cases where we are unable to definitely locate the growth, exploratory operation seems to be justifiable when the hopelessness of the case is considered. The opening of the cranium frequently relieves the suffering of the patient by reducing the intra.cranial pressure.

Brain tumors which can be diagnosed and located in the accessible parts of the cerebral cortex should be exposed by operation after a thorough course of medication. If the tumor is circumscribed, hard and superficial. the prospects for a cure are good. In operating upon cysts it is necessary to remove its walls to prevent recurrence. Filling the cavity resulting from operation with goldfoil, as advised by Estes (Journal of the American Medical Association, 18:6) has not been sufficiently practiced to render its general use advisable.

Epilepsy.-Two forms of epilepsy are amenable to operative treatment: Jacksonian and traumatic. General epileptic attacks, unaccompanied by a definite premonitory aura and in which the convulsion does not begin in any particular group of muscles, are generally not amenable to operative treatment, since one of the preliminary requisites to successful brain surgery, definite location of the lesion, is absent.

When the convulsion uniformly begins with a spasm in a distinct group of muscles or adjacent groups, trephining is a justifiable procedure. Any lesion which causes stimulation or compression of any area of the cerebral cortex may cause epilepsy. The various forms of meningitis, cysts, new growths, local. ized hemorrhage, arterio-venous aneurism, circum. scribed encephalitis, and bone fragments are the prin. cipal sources of irritation. The large majority of these may be traced to some trauma, although there may be no external evidences of it. In addition to these cases, resulting from some local disense, we find a class which results directly from trauma. Wounds of the head are much more frequently followed by epilepsy than wounds of other parts of the body. This is especially noted where there has been injury and depression of the skull which has been expectantly treated. A cicatrix of the scalp may be the source of sufficient irritation to produce epilepsy, in which case its removal may effect a cure.

In cases where the location of the trauma can be definitely located, but in which the epileptic attack begins in another region, it is best to expose the focal area involved, and if no lesion is found the point of injury can then be trephined. It will frequently be practicable to expose both areas in the same opening. Some surgeons also believe in trephining at the point of the trauma or depression, even when the attacks are not focal but general in character. This is especially feasible where the trauma is located in some region the function of which has not as yet been determined. In those cases of focal epilepsy in which no lesion is found on exploring the brain, it is advised by some operators to ascertain by faradic stimulation the exact point from which analogous movements originate and to excise it. The resultant paralysis gradually disappears if the area removed has been small.

The results of operations for epilepsy have not been as encouraging as we had reasons to hope for. Starr explains this fact upon the theory that the disease of the brain can not always be eradicated, and that while we can break up adhesion and remove scar tissue, the bealing process results in the formation of new adhesions and new scar tissue; the new conditions resulting in the return of an epilepsy which may for a time have been apparently cured. Many operators seek to prevent the adhesion of brain to meninges and bone by the interposition of a sheet of gold foil (Park). Abbe and others have used rubber tissue successfully.

The writer, not long since, operated upon a patient for the closure of a bone defect resulting from a former trephining, for epilepsy, in which several strips of rubber tissue had been interposed between the membranes. The individual strips were found com. pletely surrounded by scar tissue and the ends of two had been transposed to the outer side of the skull. Had a large single piece been used instead, the scar tissue would undoubtedly have been less, and the adhesion to the periosteum more likely prevented.

Indiscriminate operating upon the brain for epilepsy should be discouraged, but, on the other hand, in recent cases of traumatic epilepsy with distinct focal symptoms, the operation is not only admissible but should be strongly advised. The earlier the operation after the development of epilepsy the better the prospects for cure.

The statistics concerning a procedure, the technique of which is being constantiy improved, are of buit little value, except when confirmatory in character. Operations for epilepsy during the past few years are more successful than those made in preceding years. So many cases have been reported in the various standard medical journals, by reputable surgeons, that there can be no question but that many cases have been permanently cured, and as it is yet impossible to differentiate, without operation, between the various pathologic lesions, and especially since the mortality of trephining is very small and the number of recoveries on the increase, the operation is surely a justifiable one in proper cases.

Hydrocephalus.- Tapping and draining the ventricles through a trephine opening has been carefully considered by Keen, who was the first to tap the ventricle in this country, and others (Medical News, 1888). The object of the procedure being to relieve symptoms due to marked intra-cranial pressure, its scope is necessarily very limited. It may result in the cure of a primary case of hydrocephalus, as in Broca's case ("Brain Surgery," Starr) and that of Parkin (Lancet, No. 3664). As a palliative procedure it may be occasionally indicated during the course of a cerebral tumor, etc. When indicated it should be performed through a small trephine opening placed one and onefourth inches above and one and one-fourth inches behind the external auditory meatus. A trocar should be introduced and a capillary or tubular drain inserted.

Imbecility and microcephalus.-Operations for the relief of these conditions have been performed with some degree of success; serious symptoms have been relieved and in some cases cerebral development has apparently been stimulated. In cases of arrested 
development as from the presence of clots, cysts or tumors, or where premature ossification of sutures has occurred, operation may improve the condition of the patient. It should never be performed in patients over 8 years of age or in those especially weak. The cases in which an operation is justified are very rare. A sufficient number of cases have now been subjected to operation to definitely settle the fact that most of these unfortunate patients are beyond the relief of surgical treatment. An occasional brilliant success will, however, keep the profession awake to its value and, even admitting the hopelessness of this class of cases and the dangers of the operations, it is certainly a proper procedure in a small select class of cases. While Park and others believe that if one in twenty are rescued it is to be advised, Senn and others believe the procedure unwarrantable. The operationcraniotomy or craniectomy-should be extensive but preferably on one side at a time. Park prefers elevating the scalp and pericranium at one time and on a subsequent day removing the strip of bone and corresponding periosteum; this plan shortens the operation and lessens the mortality from shock, etc.

Insanity.-In cases in which mania has followed a definite trauma, which can be positively located, and in which the symptoms do not improve within a reasonable time and under appropriate medical treatment, the patient should be subjected to an explora. tory operation in the hope of finding some removable cause for the symptoms presented.

Trifacial neuralgia.-This subject is merely mentioned because the removal of a part of the Gasserian ganglion requires the opening of the calvarium. The uniformity with which sections of the distributing branches of this nerve are followed by a return of the symptoms had induced some operators to advocate the removal of the Gasserian ganglion as a primary operation. As yet, this is not a justifiable procedure, but as a secondary operation for the treatment of inveterate, recurring tic douloureux it is certainly warranted. The basal method of Rose has been superseded by the Krause-Hartley osteoplastic flap operation. The ganglion can be readily reached by an opening in the temporal region, which admits of elevation of the temporal lobe. It is impossible to remove the first division of the nerve without injuring the cavernous sinus and adjacent nerves. The other divisions can be divided and removal of much of the ganglion by divulsion readily effected. The procedure is very difficult and the mortality is large, but in severe cases it should be advised. Nerve union by regeneration has been prevented by $\mathrm{Abbe}$ by the interposition of a small piece of aseptic rubber tissue ("Annals Surgery," Vol. xxv).

Operation of trephining. - This operation formerly indicated the use of the trephine only, but by common custom it now includes opening the skull by any method. It is performed for the following purposes: 1, for the relief of compression from depressed bone, blood clot, abscess or serous effusion; 2, for relief of intra-cranial irritation; 3 , for the removal of tumors; 4 , for defective development; 5 , for the removal of foreign bodies; 6 , for the removal of the Gasserian ganglion; 7 , for exploration.

In no class of operations is absolute asepsis so imperatively demanded as in brain surgery. The scalp should be prepared by shaving and thorough disinfection with soap and ether twenty-four hours before the operation, if possible, and the wearing in the meantime of a dressing of 1-2000 bichlorid solution. The parts should again be disinfected after anesthesia has been produced. Chloroform preceded by the hyperdermic administration of a liberal dose of morphin should be preferred to ether, as it causes less cerebral stimulation and congestion. One of the essentials to successful operative work is the location of the important centers on the surface of the scalp. The fissures of Rolando and Sylvius should be carefully ontlined on the surface of the scalp and the points should be indicated on the bones by the punctures of a drill. The fissure of Rolando is outlined by a line three and one-half inches long. drawn at an angle of 67 degrees to the median line, from a point 56 per cent. of the distance back ward from the glabella (root of nose) to the inion (external occipital protuberance). For practical purposes the line begins one-half inch behind the point midway between the root of the nose and the occipital protuberance. This line, with the proper angle, can be readily outlined by folding a piece of paper so as to make an angle of 45 degrees, and then 22.5 degrees which, added together, makes the requisite angle of 67 degrees. The fissure of Sylvius is located in a line drawn from a point one and one-fourth inches behind the external angular process to a point three-fourths of an inch below the most prominent point of the parietal bone. For general purposes the line drawn from the external angular process to a point three-fourths of the distance backward from the root of the nose to the occipital protuberance will make the line of the Sylvian and the external occipito-parietal fissures, the fissure of Sylvius beginning one and one-fourth inches behind the external angular process. Reid's base line is drawn from the inferior margin of the orbit through the external auditory meatus. It is frequently utilized in locating the above-mentioned fissures. The incision should invariably be of the horse-shoe shape with the base downward toward the source of blood supply; the same to be modified as necessity may indicate. The opening in the skull can be made with a trephine and enlarged if necessary by the use of a rongeur forceps, a propelled circular saw or by the chisel and mallet. Each has its special indications and use, but the use of the chisel and mallet is undoubtedly increasing in favor. The special advantages of this method are the ready accessibility of the instruments; the method permits of making any shape and size of section; admits of preserving the nutrition of any sized section; is easy of performance and is a safe method, being practically free from danger. The dura should be opened at least onefourth inch from the edge of the bone so as to admit of suturing latter. Hemorrhage from the diploe can be controlled by crushing, by plugging with decalcified bone or with Horsley's aseptic wax. Hemorrhage of the pia mater can be controlled by clamps, ligature of vessels, or pressure with gauze compresses. Park advocates the use of $\tilde{5}$ per cent. solution of antipyrin. The brain can be safely explored with the exploring trocar. Incisions in the brain should be made with the scalpel. Cavities should be filled by gauze packing. Adherence of the brain to the dura or of dura to superficial scar can be prevented, it is claimed, by the intervention of gold foil, as advised by Beech of Boston, or by the use of rubber tissue, as practiced by Abbe, or of a thin celluloid sheet, as employed by McCosh ("Annals Surgery," Vol. xxii). The opening in the skull can be closed by various 
methods. A trephine opening can be closed with the removed button properly supported, or by the implan. tation of small fragments of bone, or bone saw-dust, or the outer table of another area of the skull can be loosened, but left attached to the periosteum and transposed to cover the defect (Nicoladoni; "Ann. Surgery," Vol. xxiii). Bone chips can be obtained by the use of the chisel or gouge on the adjacent sound bone, and should be placed on the dura, as a mosaic, with the outer side downward. These plans are known as autoplasty. By heteroplasty is meant the insertion of a piece of foreign material, as celluloid or aluminum. All of these methods are valuable and the circumstances will necessitate the use of each at times. In the replacing of large and numerous fragments after extensive fractures of the skull I have repeatedly made a satisfactory support, by making a frame work of catgut strands, which are placed in the periosteum loosely, forming a mesh not unlike loose darning. Undue pressure and unevenness can thus be readily prevented. The indications and conditions of each case must decide the question as to the use of drainage. Where there is doubt it is best to leave the wound open or provide for the free exit of blood, etc., by drainage.

1008 Fisher Building.

\section{ORIGINAL ARTICLES.}

\section{OBSTRUCTION OF THE BOWELS.}

Presented to the Medical Association of Missouri, May 20, 1897. BY T. F. PREWITT, M.D.

PROFESSOR OF PRINCIPLES AND PRACTICE OF SURGERY, MISSOURI MEDICAL COLLEGE; SULGEON TO ST. JOHN'S HOSPITAL, ETC. ST. LOUIS, MO.

"Obstruction of the bowels" is a technical phrase which implies a mechanical obstacle to the onflow of the intestinal contents. Its causes are various and the obstruction may be complete or partial, acute or chronic. It may occur at any portion of the intestinal tract from the duodenum to the rectum. It may be due to conditions that are congenital or may be acquired. But whatever may be the character of the obstruction the one striking fact dominates all consid. erations relating to treatment-it is a mechanical obstacle. A simple reference to the schedule of causes, revealed by postmortems, establishes this fact and demonstrates as well the utter uselessness of medical measures.

It is impossible here to more than outline the symptoms, diagnosis and treatment of obstruction of the bowels, acute and chronic. It is neessary to deal with these two groups separately.

In the classification of intestinal obstruction it is necessary to take into consideration both the pathologic anatomy and clinical aspects of the subject and study the relations of one to the other.

A careful collection of a large number of cases examined postmortem has shown that one of the following causes is responsible, with rare exceptions, for every case of obstruction with which we meet: 1 , internal hernia: 2 , twists (volvulus) ; 3 , bands of some kind; 4, fecal impaction; 5, mechanical pressure of tumors; 6 , stricture; $i$, contractures due to matting together of intestinal coils from peritoneal and cancerous diseases; 8 , intussusception; 9 , gall stones; 10 , enteroliths; 11, foreign bodies. A more practical classification, however, is that in which all cases are divided into acute and chronic.
A study of the pathology in connection with the clinical history reveals a striking relationship between the causes and the group of symptoms which are asso. ciated with them. Thus, of the causes leading to acute obstruction we have: 1, internal hernia: 2 , twists (volvulus); 3 , bands: 4 , intussusception; $\tilde{5}$, gall stones. Of the leading causes of chronic obstruction we have: 1, fecal impaction; 2, mechanical pressure of tumors; 3 , stricture; 4 , contractures; 5 , intussusception, which may be and is sometimes chronic.

So constant is the relationship between primarily acute obstruction and the causes grouped above that we can say that any given case is due to one of these, and the same may be said of primarily chronic cases. Symptoms.--In acute cases these come on suddenly in a previously healthy indiviclual and are striking and characteristic. The attack is sudden and acute. Pain is paroxysmal, central and fixed. Vomiting sets in early, first of the contents of the stomach, then of the duodenum, then of the small intestines and rapidly becomes fecal. The patient becomes faint, pale and markedly collapsed; constipation is absolute, not even flatus escaping from the bowels. Distention of the abdomen rapidly takes place and distended coils of intestines may often be both seen and felt. The urine may be scanty or even suppressed.

It will be seen that these are the symptoms of acute strangulated hernia. And why not? There is the same interruption to the onflow of the intestinal contents, the same obstruction to the blood current; the same disturbing and depressing effect on the nervous system, the same violent peristaltic action in the endeavor to overcome the obstruction and push on the obstructed contents, the same paroxysmal pains and eventually the same fatal result from precisely the same causes.

When, therefore, we are confronted with a case presenting such a group of symptoms there can be no. doubt as to the diagnosis. A dire disaster has befallen the patient. Death confronts him and only the coolness, the courage and skill of the surgeon can rescue him from its grasp.

The bowels do not move. Shall we give purgatives? Already the bowel is making frantic efforts to force the blockade. Shall we stimulate it to more furious efforts to accomplish the impossible? No. It would be gross malpractice.

The patient suffers great pain. Shall we give opium? If it be used only as an adjuvant to annul pain, to relieve collapse, to restore the action of the kidneys, if it is administered for these purposes only while the surgeon prepares to relieve the strangulation, yes. If it is given with any curative purpose, then emphatically, no. We do but invite euthanasia. The patient relieved of his suffering is deluded with the idea that he is doing well, the doctor no longer assailed by the cries of the patient, too often hesitates and the golden moments slip by, during which the patient might be rescued from an otherwise inev. itable doom. When the diagnosis is once made every hour of delay is compromising his safety. The bowel is damaged, peritonitis sets in, collapse ensues and the patient dies in spite of the delayed operation.

When the diagnosis is made celiotomy is the only thing consistent with that condition. If there be any doubt an exploratory celiotomy should be done to remove that doubt.

It is scarcely worth the time to discuss certain measures that are advised in these cases. Of what use is. 